\title{
Crossed sagittal-suture cephalhematoma
}

\author{
Paul Merlob • Gil Klinger
}

Received: 2 January 2009 / Published online: 11 February 2009

(C) Springer-Verlag 2009

\section{Dear Editor:}

We read with great interest the article by Kaiser and Oesch entitled "Sagittal craniosynostosis combined with ossified cephalhematoma - a tricky and demanding puzzle" published in your journal [1]. The authors state that "midline vertical site is quoted only once in the literature". However, we have previously published, in the British Journal of Radiology a paper entitled; "Crossed sagittalsuture cephalhematoma" [2]. We described a 3,500-g baby girl delivered by forceps at 40 weeks gestation. On physical examination, a midline palpable skull prominence was seen. At the age of 4 days, skull radiography showed a partial sagittal craniosynostosis and soft tissue swelling compatible with a cephalhematoma overlying the craniosynostotic section of the sagittal suture. A follow-up X-ray at 7 weeks showed that the soft tissue mass seen at birth was circumscribed by a well-calcified rim extending over both parietal bones. The final diagnosis was a calcified cephalhematoma overlying a sagittal craniosynostosis. We concluded that a cephalhematoma can be located in the midline over a suture only in the presence of sagittal craniosynostosis.

The prevalence of crossed sagittal-suture cephalhematoma in our newborn population is extremely rare. In our Department of Neonatology Registry of Congenital Anomalies affiliated with the International Clearinghouse for Birth Defects Surveillance and Research from 1974, the present case was the only one found in 154,389 live births.

\section{References}

1. Kaiser GL, Oesch V (2009) Sagittal craniosynostosis combined with ossified cephalhematoma - a tricky and demanding puzzle. Childs Nerv Sys 25:103-110

2. Merlob P, Grunebaum M, Reisner SH (1985) Crossed sagittal-suture cephalhematoma. Brit J Radiol 58:1007-1008

P. Merlob · G. Klinger $(\bowtie)$

Department of Neonatology,

Schneider Children's Medical Center of Israel,

14 Kaplan St,

Petah Tiqva 49202, Israel

e-mail: gilk1@post.tau.ac.il

P. Merlob $\cdot$ G. Klinger

Sackler School of Medicine, Tel Aviv University,

Tel Aviv, Israel 